\title{
Gastrobronchial fistula and anastomotic esophagogastric stenosis after esophagectomy for esophageal carcinoma
}

\author{
R. Aguiló Espases, MD, PhD, R. Lozano, MD, PhD, A. C. Navarro, MD, F. Regueiro, MD, E. Tejero, MD, PhD, and \\ J. C. Salinas, MD, PhD, Zaragoza, Spain
}

$\mathrm{E}$ sophagectomy and esophageal replacement with a gastric tube are the procedures of choice for resectable esophageal carcinoma. Postoperative stricture at the level of the esophagogastric anastomosis is a wellknown complication of this procedure. ${ }^{1,2}$ Much less common is the development of a benign fistula communicating the gastric tube and the airway. ${ }^{3,4}$

We report the clinical case of a patient in whom successful surgical repair of the 2 complications was simultaneously achieved in a single-staged operation.

\section{Clinical Summary}

A 51-year-old male patient had epidermoid carcinoma of the middle third of the esophagus. After neoadjuvant chemotherapy and radiation therapy, he underwent total esophagectomy and mediastinal lymphadenectomy through a right-sided thoracotomy. The esophagus was replaced with a gastric tube, and the esophagogastric anastomosis was performed manually in the neck. Postoperatively, the patient had external anastomotic leakage that was managed with full fasting and enteral nutrition. Closure of the leakage ensued. However, a circumferential, cicatricial stenosis developed at this level. Repeated endoscopic dilations resulted in recurrent restenosis. Finally, an auto-expandable metallic "wallstent" prosthesis was left in place in an attempt to provide sufficient lumen and improve swallowing.

Some months later, the patient returned with severe hemoptysis and dysphagia. Esophagography showed an "H"-type bronchogastric fistula (Figure 1). Severe stenosis at the esophagogastric anastomosis was also present. Computed tomography (CT) of the thorax showed that the prosthesis had moved downward and had made an impact against the anterior wall of the gastric tube, at the level where it crossed the origin of the right main bronchus (Figure 2). Before any surgery was planned, local recurrence of the tumor was ruled out by pathologic examination of biopsy samples obtained endoscopically from the margins of the fistula. A wholebody CT scan was performed to exclude distant metastases.

\footnotetext{
From the Servicio de Cirugía General y Torácica "A," Hospital Clínico Universitario "Lozano Blesa," Zaragoza, Spain.

Received for publication July 11, 2003; accepted for publication Aug 11, 2003.

Address for reprints: Rafael Aguiló Espases, Servicio de Cirugía General y Torácica "A,” Hospital Clínico Universitario "Lozano Blesa,” P./San Juan Bosco, 15, Zaragoza 50009, Spain (E-mail: cgah@hcu-lblesa.es).

J Thorac Cardiovasc Surg 2004;127:296-7

$0022-5223 / \$ 30.00$

Copyright (C) 2004 by The American Association for Thoracic Surgery

doi:10.1016/j.jtcvs.2003.08.009
}

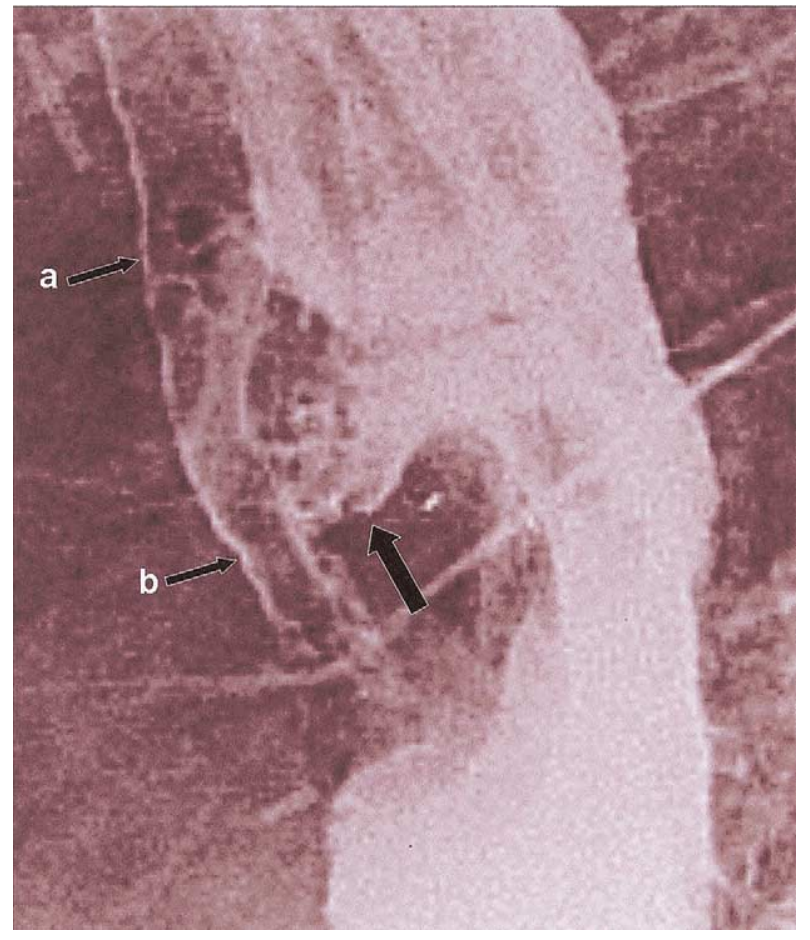

Figure 1. Preoperative esophagogram. An "H"-type fistula (large arrow) communicating the gastric tube and the airway (small arrows) is shown.

Surgical repair was performed through a right-sided thoracotomy. The fistula was dissected and severed. Manual suturing of the bronchus wall and of the gastric defect was carried out. The sutures were reinforced by interposing a vascularized intercostal myoplasty. The prosthesis was removed through a gastrotomy. The esophagogastric stenosis was transacted with an end-to-end anastomosis automatic stapler, which was introduced into the gastric tube lumen through the gastrotomy, while the anvil was passed through the mouth and pharynx. In the early postoperative period, the patient had to be rethoracotomized due to hemothorax. Afterward, normal recovery ensued. Oral intake could be reinitiated on the tenth postoperative day. Six months later, the patient retains satisfactory oral feeding status and is in good general condition.

\section{Comments}

A benign fistula between the airway and the gastric tube replacing the esophagus after total esophagectomy is very rare but potentially fatal. ${ }^{3,4}$ It may develop most often secondary to leakage of the anastomosis with inflammatory involvement of the tracheo- 


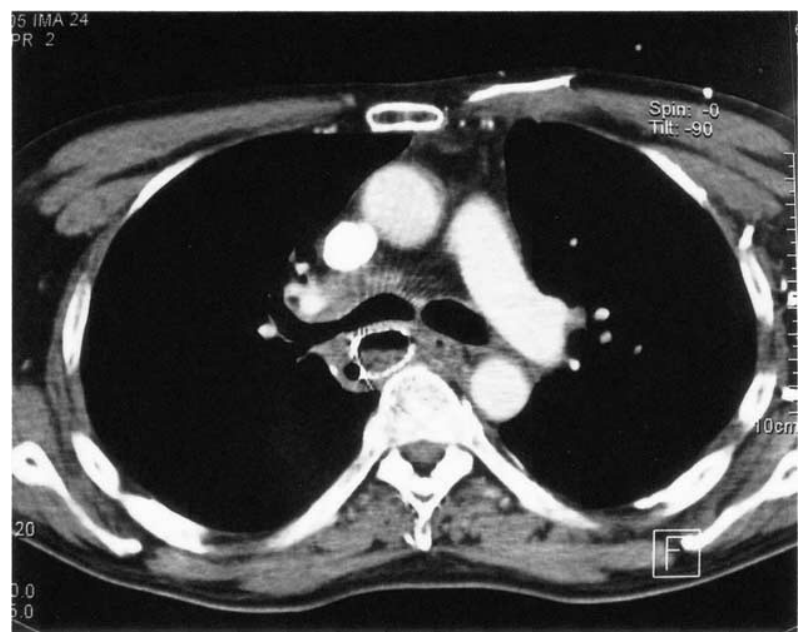

Figure 2. Preoperative CT scan. The metallic wall-stent prosthesis compresses the anterior wall of the gastric tube against the "pars membranacea" of the right main bronchus.

bronchial tree or after endoscopic dilatation of a benign anastomotic stricture. Other causative factors have only been reported as solitary cases. ${ }^{4}$ Among these, we have found in the literature just 1 other case in which the tracheogastric fistula presented in association with an auto-expandable esophageal wall-stent prosthesis. ${ }^{5}$

Symptoms at presentation may range from mild to life-threatening. ${ }^{3,4}$ Yet the possibility of a rapid deterioration of the patient's general condition should always be kept in mind. Just on suspicion, a barium esophagogram should promptly be performed for diagnosis. Treatment is always challenging and has to be individually tailored. It will depend on the severity of symptoms, on the size and location of the fistula, and on accompanying conditions. If surgery is required, the procedure of choice is excision of the fistula and closure of the tracheal and esophageal defects. Interposition of a pedicled pleural, omental, or muscle flap has proved to be useful in preventing recurrence of the fistula. The gastric tube should be left in place unless judged as an unviable option. In such case, colonic interposition is indicated to restore the continuity of the gastrointestinal tract. If mediastinitis is present, elimination of the septic focus and extensive drainage of the mediastinum are mandatory. ${ }^{3,4}$

\section{References}

1. Jeyasingham KJ. Benign strictures of the esophagus. In: Shields TW, LoCicero J III, Ponn RB, editors. General thoracic surgery. 5th ed. Philadelphia: Lippincott Williams \& Wilkins; 2000. p. 1865-80.

2. Law S, Fok M, Chu KM, Wong J. Comparison of hand-sewn and stapled esophagogastric anastomosis after esophageal resection for cancer. A prospective randomised controlled trial. Ann Surg. 1997;226: 169-73.

3. Gudovsky LM, Koroleva NS, Biryukov YB, Chernousov AF, Perelman MI. Tracheoesophageal fistulas. Ann Thorac Surg. 1993;55:868-75.

4. Buskens JC, Hulscher JBF, Fockens P, Obertop H, van Lanschot JJB. Benign tracheo-neo-esophageal fistulas after subtotal esophagectomy. Ann Thorac Surg. 2001;72:221-4.

5. Schowengerdt CG. Tracheoesophageal fistula caused by a self-expanding esophageal stent. Ann Thorac Surg. 1999;67:830-1.

\title{
Bipulmonary transplants with lungs obtained from two non-heart- beating donors who died out of hospital
}

\author{
José Ramón Nuñez, MD, PhD, ${ }^{a}$ Andrés Varela, MD, PhD, ${ }^{\mathrm{b}}$ Francisco del Río, MD, ${ }^{a}$ Pablo Gámez, MD, ${ }^{b}$ \\ Joaquín Calatayud, MD, ${ }^{a}$ Mar Córdoba, MD, ${ }^{\text {b }}$ Florentino Hernando, MD, ${ }^{a}$ Piedad Ussetti, MD, ${ }^{\mathrm{b}}$ Ana Gómez, MD, \\ Maria Cruz Carreño, MD, ${ }^{\mathrm{b}}$ Antonio Torres, MD, PhD, ${ }^{\mathrm{a}}$ Javier Gómez, MD, ${ }^{\mathrm{b}}$ José Luis Balibrea, MD, PhD, and \\ Ana López, MD, ${ }^{\mathrm{b}}$ Madrid, Spain
}

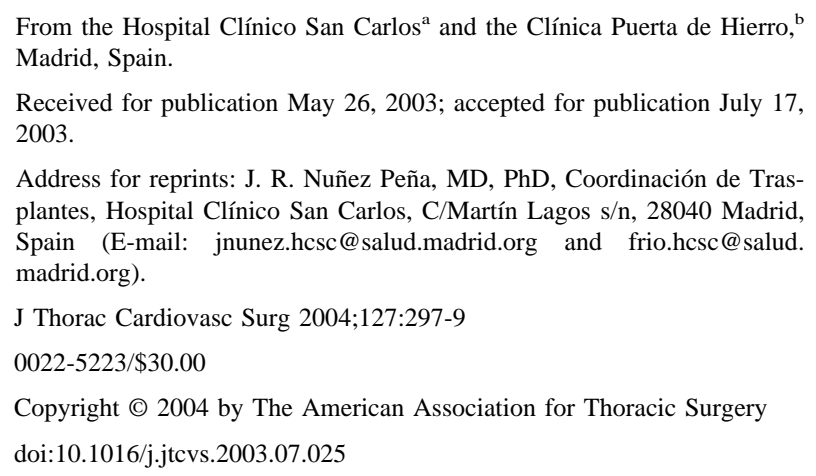

I $\mathrm{n}$ Madrid there is a specific protocol (code 9) that allows out-of-hospital emergency service personnel and transplant teams of Hospital Clínico San Carlos (HCSC) to harvest organs from non-heart-beating donors (NHBDs) for transplantation. We have reported on the quality of kidneys obtained from NHBDs. ${ }^{1,2}$ On the basis of the first medical report of a lung transplant from an $\mathrm{NHBD},{ }^{3}$ we conducted a clinical, functional, and histologic study in collaboration with the Thoracic Surgery Service of Clínica Puerta de Hierro to prove the quality of lungs from NHBDs.

\section{Clinical Summary}

After 30 minutes of cardiopulmonary resuscitation maneuvers are performed in the potential donor, the medical staff assesses cardiac 